\title{
Tissue Integration of Synthetic Grafts and the Impact of Soft-Tissue Infection - An Experimental Model
}

\author{
Russu Eliza1*, Mureșan Adrian Vasile ${ }^{1}$, Cordoș Bogdan Andrei², Cotoi Ovidiu Simion³, Copotoiu \\ Constantin $^{1}$
}

\author{
1 Department of Surgery, University of Medicine and Pharmacy of Tirgu Mures, Romania \\ 2 Experimental Research Station, University of Medicine and Pharmacy of Tirgu Mures, Romania \\ Pathophysiology Department, University of Medicine and Pharmacy of Tirgu Mures, Romania
}

\begin{abstract}
Objective: Starting with the 'Vinyon-N-revolution' of the 50's, there has been a constant interest in understanting tissue integration, or the so-called graft healing process, as well as its relationship with infection. In this study we present an experimental animal model designed to assess tissue integration of different graft materials, and their reaction to the presence of infection. Methods: Synthetic grafts (knitted Dacron $\AA$, woven Dacron ${ }^{\circledR}$, silver-impregnated Dacron $®$ and Gore-Tex $®$ ) were implanted subfascially in the interscapular region of Wistar rats. Animals were divided into a control group and an infected group, with infection induced using bacterial suspensions of standard strains of Staphylococcus aureus, Staphylococcus epidermidis, and Escherichia coli. Implants were retrieved at 2 and 4 weeks postoperatively in the control group and at 1, 2 and 3 weeks postoperatively in theinfected group. Retrieved grafts were assessed bacteriologically and morphopathologically. Results: All microorganisms produced clinically evident infections, with positive blood cultures in case of E. coli. Staphylococci produced more massive infections on Dacron ${ }^{\circledR}$ grafts, except for the silver-impregnated version, while E. coli produced more significant infections on Gore-Tex® grafts. Morpho-pathologically Dacron ${ }^{\circledR}$ grafts behaved poorly, with ocassional complete structural compromise, and no difference between the conventional and the silver-impregnated type. The Gore-Tex® graft showed a consistent structural resistance throughout the study period. Conclusions: Although the silver-impregnated graft inhibited bacterial growth, it was poorly tolerated by the host tissue. In contrast, Gore-Tex® grafts showed more massive infection, especially with E. coli, but kept their structural integrity surprisingly well.
\end{abstract}

Keywords: vascular grafts, silver impregnation, rats

Received: 14 August 2015 / Accepted: 03 September 2015

\section{Introduction}

Since the emergence of the first textile grafts, scientists have been trying to get a better understanding of the process of "graft healing"[1]. Technological progress has lead to the design of materials like polyethylene terephthalate (Dacron $^{\circ}$, woven or knitted) [2] and expanded polytetrafluoroethylene (ePTFE or Gore-Tex ${ }^{\circ}$ ), which proved to be the most favorable in terms of durability and thrombogenicity. Both materials have been improved over time, especially in terms of porosity and the addition of special coatings such as anticoagulants, silver acetate or antibiotics (rifampicin).

First introduced in 1939, Dacron ${ }^{\circledR}$ is a polymer available in two forms, a knitted and a woven one. In its woven form, Dacron ${ }^{\oplus}$ multi-filaments are arranged in an overlapping pattern both lengthwise and circumferentially, rendering the graft difficult to incorporate into the tissues. In the knitted form of the material, fibers are arranged in an intercalated, continuous pattern, and positioned in a longitudinal but not a circumferential direction. The technique of external velouring was also developed in an effort to enhance tissue incorporation of grafts [3]. The process of crimping can increase graft flexibility, but reduces its internal diameter [4] and creates inequalities of the lumen. Knitted Dacron ${ }^{\ominus}$ grafts are characterized by high porosity, which brings about the need for pre-clotting. Manufactur-

* Correspondence to: Eliza Russu

E-mail: eliza.russu@umftgm.ro ers use either gelatin, collagen or albumin to seal the pores, possibly fixed by formaldehyde crosslinking (which allows collagen to be absorbed by the body within 2 weeks), or the more efficient glutaraldehyde crosslinking (applied for albumin, which will be degraded in approximately 2 months). In the most recent versions, aldehyde- and isocyanate free impregnation uses dehydrothermal crosslinked collagen (Bioseal ${ }^{\circledR}$ technology).

Teflon was the name under which ePTFE was first introduced in 1937. It is an extruded polymer, expanded by heating and stretching, which has a non-textile characteristic, with a "node-fibril"-type pattern. Porosity is defined by the internodal distance, which in this case is of $30 \mu \mathrm{m}$. Attempts were made to manipulate tissue integration of ePTFE by increasing the internodal distance, and thus stimulate transmural tissue growth. Other changes have also been made in an effort to lower the material's thrombogenicity by creating an electronegative luminal surface (through carbon coating), or heparin impregnation [5]. Silver acetate impregnation [6] is employed for its antimicrobial effect, combined with collagen impregnation [7]; however the efficacy is lower compared to that of impregnation with rifampicin. Some silver-impregnated prostheses have a special design that permits soaking in a solution of rifampicin [8].

Polyurethane [9] is the third type of synthetic material used for vascular grafts, however its use is controversial. The elastic and compliance properties, as well as the inertia 
of the material are unique and superior compared to other materials. Nonetheless, once implanted, its degradation products have been shown to posses a potential carcinogenic effect, which determined many producers to further test the material. Meanwhile hybrid products [10] are also emerging, together with innovative materials created by genetic bioengineering [11]; clinical trials are underway to demonstrate the possible benefits of these products.

One of the major complications of synthetic implants is infection [12,13], with an incidence varying between 0.2 and $5 \%$, depending on the type of implant, its location, and other characteristics of the host [14]. The most commonly used clinical classification of graft infections (also applicable for postoperative wound infections in general) is the Szilagyi classification, in which grade 1 is defined by cellulitis involving the postoperative wound, grade 2 refers to an infectious process that includes the subcutaneous tissue, and grade 3 implies the actual involvement of the vascular prosthesis.

The specialty literature confirms that, from a bacteriological perspective, graft infections are predominantly caused by Staphylococcus aureus [15], which generates between $25 \%$ and $50 \%$ of cases. Staphylococcus epidermidis and Candida albicans are responsible for many cases that present with negative cultures and usually have a characteristic late clinical expression (over 4 months post-implantation). There has also been and increase in cases of severe infection caused by Escherichia coli, which rapidly cause anastomotic dehiscences at the sites of graft implantation. In addition, infections caused by methicillin-resistant Staphylococcus aureus (MRSA) already represent 25\% of early graft infections (occurring in less than 4 months post-implantation). The rapid and worrying increase in the incidence of infections with resistant organisms like MRSA justifies the prophylactic use [16] of grafts with a better capacity of defense such as the antibiotic- or silverimpregnated types [17] in selected cases.

An animal model of tissue incorporation and changes thereof in the presence of infection is, in our opinion, useful in terms of guiding the appropriate and differentiated clinical use of a particular type of prosthesis, depending on the special requirements of each particular case. The aim of this study was the development of such a model using the most common graft materials and the most frequently encountered infectious organisms.

\section{Methods}

A number of 120 Wistar rats were used, 4-5 months of age, each weighing approximately 500 grams. The animals were purchased from the Cantacuzino Institute Experimental Station, Bucharest, and divided into 2 groups: a control group and an infected group. The rats were housed in groups of 5 in special polycarbonate cages as per European standards, and were kept in quarantine for one month prior to surgery, for acclimatization; they were fed with combined granulated chow and water ad libitum. The study was approved by the Scientific Research Ethics Commitee of the University of Medicine and Pharmacy Targu Mures, decision number 73/ 20.07.2015.

\section{Graft implantation and infection}

The animals were anesthetized using a combination of ketamine and xylazine $(75 \mathrm{mg} / \mathrm{kg}$ and $10 \mathrm{mg} / \mathrm{kg}$, respectively) administered intraperitonealy. A 5 by $5 \mathrm{~cm}$ surgical area was prepared in the interscapular region, and a $3 \mathrm{~cm}$ long longitudinal interscapular incision was made in the median line, after local infiltration of the incision line with $0.1 \mathrm{ml}$ adrenaline and $0.2 \mathrm{ml}$ of lidocaine, to reduce bleeding.

Following the dissection of the subcutaneous tissue and fascia, the acromiotrapezius and spinotrapezius muscles were identified, and a 2 by $2 \mathrm{~cm}$ piece of each type of synthetic prosthetic material was sutured subfascially on the muscular plane, using two polypropylene monofilament sutures. Four types of polyester prostheses were used: knitted, woven, and silver-impregnated Dacron ${ }^{\circ}$, and ePTFE Gore-Tex ${ }^{\circ}$ grafts; these were each implanted in 8 animals from the control group and 20 animals from the infection group. The fascial gap was closed with Bicril 3.0 separate vertical mattress sutures.

For the group of infected animals the surgical intervention was similar. The inocula were prepared from $1 \mathrm{McFar}-$ land stock dillutions of standard bacterial strains: methicillin-susceptible Staphylococcus aureus (MSSA) - American Type Culture Collection (ATCC) 29213 (subspecies aureus Rosenbach, strain designation: Wichita), MRSA ATCC 43300 (subspecies aureus Rosenbach, strain designation: F-182), and E. coli ATCC 25922 (Migula 1895, Castellani and Chalmers 1919, strain designation: FDA strain Seattle 1946). The $1 \mathrm{McF}$ arland standard was chosen based on the preliminary results obtained in a subgroup of 8 rats in which a $0.5 \mathrm{McF}$ arland inoculum proved to be insufficient to induce even an infection of grade 1 according to Szilagyi's classification - these animals were subsequently excluded from the study. An inoculum of $0.3 \mathrm{ml}$ was used from each strain, which corresponds to approximately $0,9 \times 10^{8}$ colony-forming unit (CFU); these were introduced with a $27 \mathrm{G}$ needle between the sutures, before tying the last suture. Each bacterial strain was used in a total of 48 animals, 6 subgroups of 8 animals each implanted with the 4 types of grafts, with the exception of MSSA infected rats, which were in total 32 animals, 4 subgroups of 8 animals each implanted with the 4 types of grafts. The explanation for this excedent is the changing of the design of the sub-groups in the attempt of a better glimpse at each infection's pattern of evolution if followed closely, within 7 days distance in retrieval.

\section{Follow-up and implant retrieval}

Animals were observed clinically for 1 month postoperatively by assessing the local evolution of the surgical wound and temperature measurements performed on postopera- 
tive days 2, 5 and 7. Blood samples were collected on days 2, 5, 7 and 14 for white blood cell count, and on days 2 and 7 for hemoculture: rats were placed in restraining tubes, the animals tails was immersed in water at $40-50^{\circ}$ $\mathrm{C}$ for 5 minutes, and $0.5-1 \mathrm{ml}$ of blood was drawn from the lateral tail vein.

For implant retrieval, animals were eutanized with 0.1 $\mathrm{ml} \mathrm{T61} \mathrm{(embutramide} 200 \mathrm{mg}$, mebezonium iodide 50 $\mathrm{mg}$, clorhidric tetracaine $5 \mathrm{mg}$ ), which stopped the heart of the rats in 10-20 seconds, administered intracardially after prior anesthesia with ketamine and xylazine. Figure 1 shows the distribution of animals based on implants, infectious organisms and implant retrieval. In the subgroup of control animals (8 implanted with each type of graft), 4 implants were retrieved each at 2 weeks and 4 weeks postoperatively.

As mentioned above, the first subgroup of 8 rats infected with MSSA was excluded from the study (it was also observed that the retrieval of the grafts after 1 month is practically impossible, as the implant is rejected by the animal's body and literally "disappeares" by expulsion from the wound). The implants from the second subgroup of 8 rats infected with MSSA were retrieved at 2 weeks postoperatively.

The rest of the animals from the infection group (24 each in the MSSA, MRSA and E. coli subgroups respectively) have had the implant retrieved at 1,2 and 3 weeks postoperatively ( 8 animals at each time point, 2 with each type of graft).

\section{Processing of retrieved implants}

Each piece of retrieved graft was cut into two halves: one was sent for seeding on media, and the other used for morpho-pathological analysis, the latter data being detailed in a different paper, submitted for publication, as part of the same reseasrch project. For the bacteriological evaluation, the graft pieces introduced in tubes with $3 \mathrm{ml}$ of saline solution were processed as follows:

The sample was vortexed for 30 seconds to detach the bacteria from the vascular prosthesis;

Serial dilutions were performed in sterile saline $(1 / 10$, 1/100, 1/1.000, 1/10.000, 1/100.000);

From each of these dilutions, $50 \mu \mathrm{l}$ were seeded on a blood agar plate (previously kept at $37^{\circ} \mathrm{C}$ )

The plates were incubated at $37^{\circ} \mathrm{C}$ for 24 hours and then, of each plate ( corresponding to a certain dilution), the formed colonies were counted using the 'IUL Flash \& Grow' automatic colony counter. The resulted numbers were adjusted for dilution and volume to obtain the number of CFU/ ml, using the following formula:

\section{$\mathrm{CFU} / \mathrm{ml}=($ no. of colonies $\mathrm{x}$ dilution factor $\mathrm{x} 20) / 3$}

$* / 3$ = adjusting for total volume;

** 20 = adjusting for the volume of the inoculum

\section{Statistical analysis}

The data were analyzed using the EpiInfo7 statistical program. For comparing the central tendencies the KruskallWallis was used.

\section{Results}

As mentioned earlier, a number of 8 rats had to be exluded from the study because the clinical infection wasn't sufficient to be relevant for the results obtained post-retrieval. It was also apparent that maintaining the infected graft for a month postoperatively was useless, as the graft pieces were totally expelled at the end of the period.

Surgical wound infection was evident in all 80 rats from the infected group (as shown in figure 2). In case of MSSA, the infection tended to reach a maximum of grade 2 according to Szilagyi's classification by 1 week postoperatively, with a consecutive reduction of the infection grade by the $21^{\text {st }}$ postoperative day. For the subgroup infected with MRSA, a Szilagyi grade 3 infection was produced early, with no tendency of reduction by 21 days postoperatively. In terms of the E. coli infection, a difference was observed in the case of both Dacron grafts and the ePTFE graft: for the former grafts, starting from an initially reduced Szilagyi grade, the infection subsequently aggravated, while in case of the latter there was a massive infection right from the start.

Rectal temperature measurements performed on days 2 , 5 and 7 postoperatively showed an average value of $39^{\circ} \mathrm{C}$ in the $2^{\text {nd }}$ postoperative day, which decreased to an average of $38.8^{\circ} \mathrm{C}$ by the $5^{\text {th }}$ day and $38.5^{\circ} \mathrm{C}$ on the $7^{\text {th }}$ day, indicating that all rats became afebrile by day 7 , irrespective of graft type.

Blood samples collected on the $2^{\text {nd }}$ and $7^{\text {th }}$ day postoperatively showed a positive blood culture on the $2^{\text {nd }}$ day only in the 24 animals infected with $E$. coli, and the samples were negative on the $7^{\text {th }}$ day. One death occurred during blood sample harvesting in an animal from the E. coli subgroup, immediately after the induction of anesthesia. The morphopathology analysis from samples taken from the lungs showed the death occurred because of acute respiratory distress syndrome.

White blood cell counts obtained from the samples collected on days 2, 5, 7 and 14 postoperatively did not vary according to the type of graft, but were influenced by the infecting bacteria. The average value on day 2 was 10.2 $10 \mathrm{e} 12 / \mathrm{L}$, increasing to $13.810 \mathrm{e} 12 / \mathrm{L}$ by day 5 , and reaching a maximum on day $7(14.410 \mathrm{e} 12 / \mathrm{L})$, with a subsequent decrease to $8.410 \mathrm{e} 12 / \mathrm{L}$ on day 14 postoperatively.

In terms of microbiological assessment, statistical analyses were made for CFUs/ $\mathrm{ml}$ obtained in case of the $1 / 100.000$ dilution for each bacterial strain. In regard to E. coli infection, a statistically significant difference was found between woven Dacron ${ }^{\oplus}$ and $\operatorname{ePTFE}(\mathrm{p}<0.05)$, with the latter showing a larger number of CFUs/ $\mathrm{ml}$. These results were not influenced by the day of implant retrieval. 


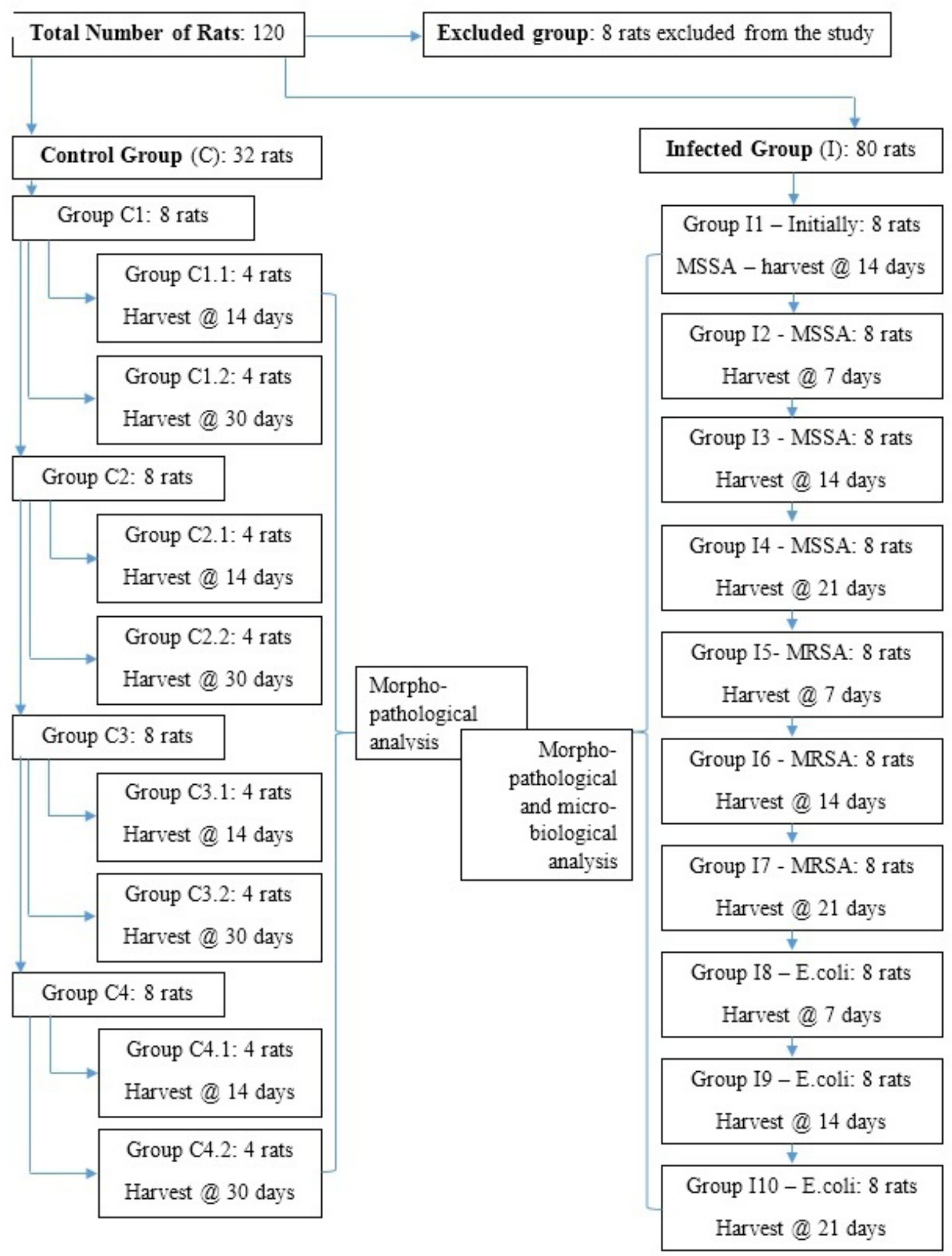

Fig. 1. Diagram showing the distribution of the rats used in the study, based on type of implant, infectious agent, and implant retrieval.

The silver-impregnated Dacron ${ }^{\bullet}$ showed the smallest number of CFUs/ $\mathrm{ml}$ in case of $E$. coli infection.

There was a statistically significant difference between the CFUs/ $\mathrm{ml}$ obtained for the 4 types of grafts in case of both MSSA and MRSA infections (figures 6 and 7): thus the ePTFE grafts had a smaller number of CFUs/ $\mathrm{ml}$ compared to knitted and woven Dacron ${ }^{\bullet}$, comparable to those obtained for the silver-impregnated Dacron ${ }^{\circ}$. For the MSSA infection, the number of CFUs/ $\mathrm{ml}$ in case of silverimpregnated Dacron ${ }^{\circledR}$ was zero.

\section{Discussion}

In the late 80 's scientists began the study of silver-impregnation of medical devices. Silver is a broad spectrum bacte- 


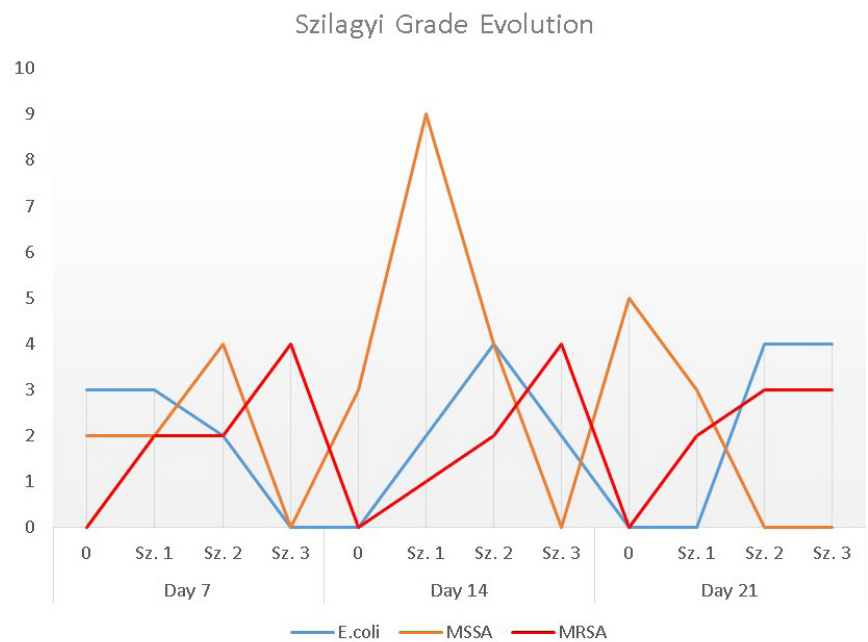

Fig. 2. The evolution of clinical infection of the surgical wound, based on Szilagyi grades (Sz. 1, Sz. 2, Sz. 3 are Szilagyi grades, Day 7, Day 14, Day 21 are the 7th, 14th and 21st day postoperatively).

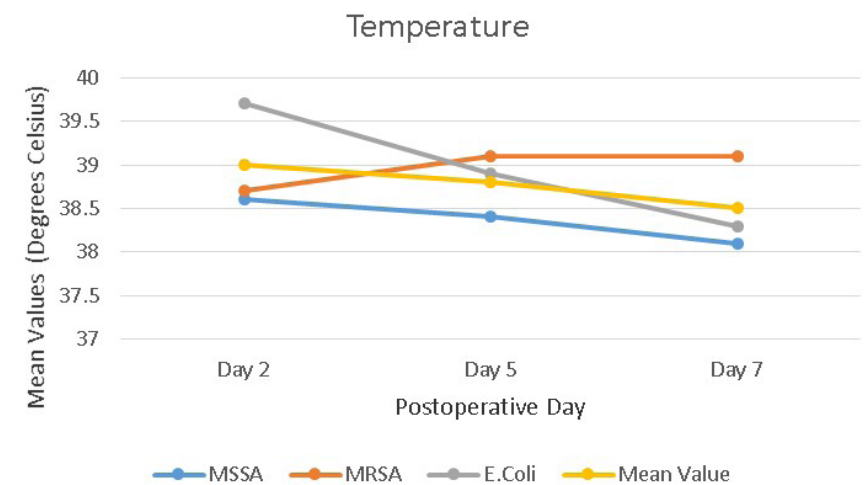

Fig. 3. The evolution of rectal temperature in the infected group

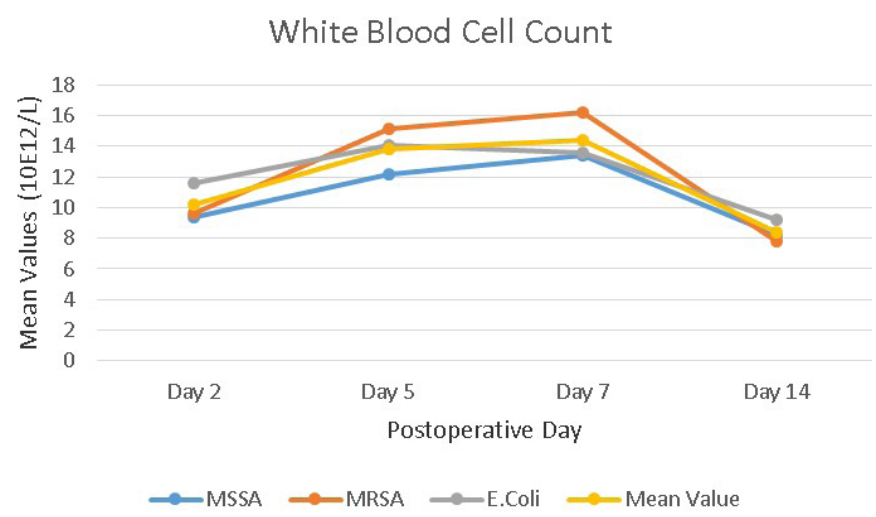

Fig. 4. The evolution of white blood cell counts in the infected group.

riostatic agent that binds to microbial DNA and prevents its replication. Silver-impregnation was used for all kinds of medical instruments: diffrent types of catheters, heart valves, suture material, bone implants, etc.

In an experimental study on dogs, Shah et al.[18] compared the results of using Gore-Tex ${ }^{\oplus}$ grafts treated with either norfloxacin alone or by norfloxacin and silver-impregnation, and found that the addition of silver yielded superior results. Benvenisty [19] conducted a similar study on dogs, demonstrating the efficacy of vascular grafts impregnated with silver.

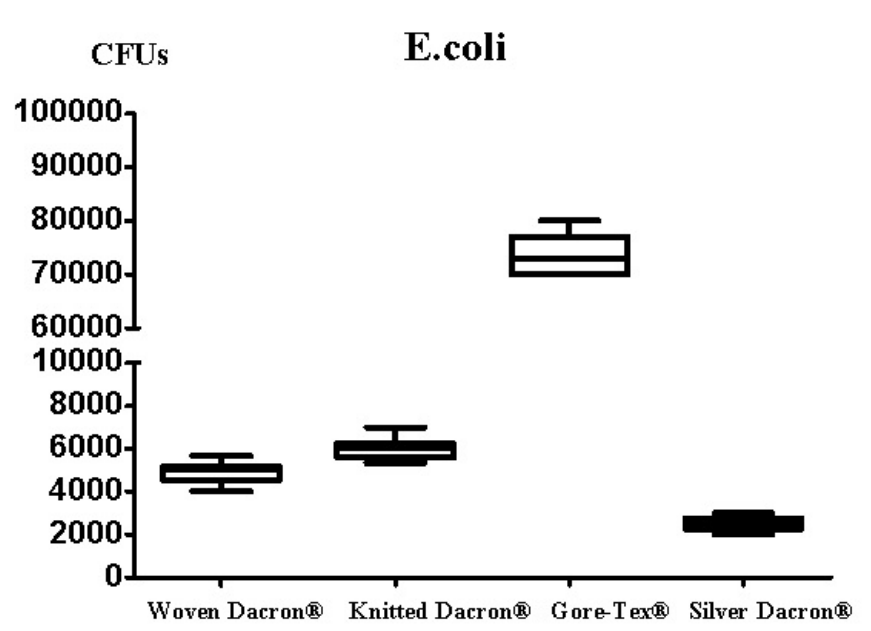

Fig. 5. The central tendencies of CFU values retrieved from each type of graft infected with E.coli

\section{CFUs MRSA}

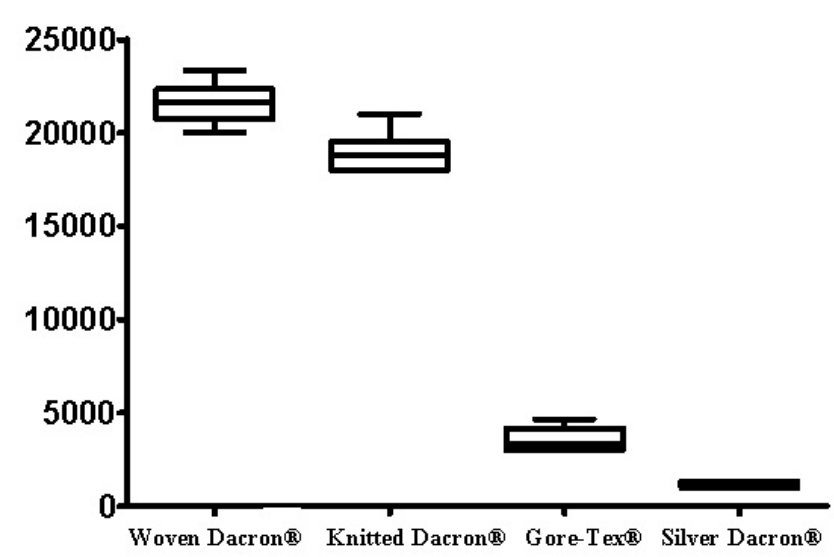

Fig. 6. The central tendencies of CFU values retrieved from each type of graft infected with MRSA

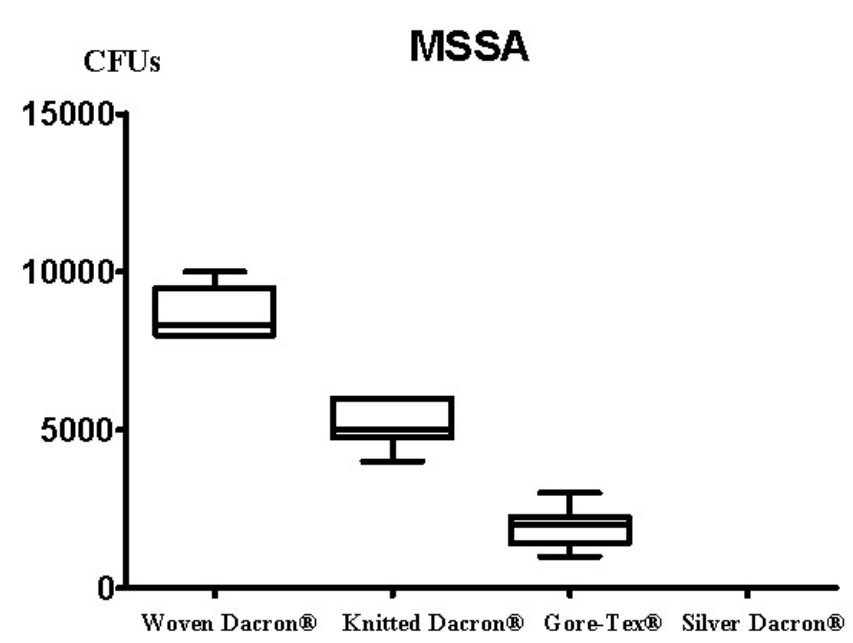

Fig. 7. The central tendencies of CFU values retrieved from each type of graft infected with MSSA

Illingworth et al. [20] and Collin [21] observed a clear reduction in infection rates following the subcutaneous implantation of a silver-impregnated cardiac valve in dogs, as well as a significant reduction in the number of bacterial contamination of central venous catheters with silver coat- 
ing. In contrast to these findings, Goeau-Brissoniere et al. [22] found no effect of the use of silver in preventing infection, in yet another experimental study on dogs.

Given that our department and most vascular surgery departments in the country are using silver-impregnated grafts, particularly in situations with increased risk of infection, and lacking a considerable experience with rifampicin-impregnated grafts, we considered it relevant to design an experimental study comparing silver-impregnated grafts with other standard grafts used in revascularization procedures. The infectious microorganisms were also chosen based on their relevance to human infections identified most commonly in our practice: MSSA, MRSA and E. coli.

After standardizing our method, we found that all the used microorganisms produced surgical wound infections that were clinically evident. In case of MSSA, the infection was limited in grade, with a tendency of healing after day 14. MRSA produced infections which were more severe from the start, with no tendency to heal. Infections produced by $E$. coli were milder in certain types of grafts, and more severe in others, also displaying no tendency towards healing; as a further difference there was a pattern of later onset compared to infections produced by Staphylococci. Blood cultures were negative for Staphylococci and positive in case of $E$. coli, regardless of the type of implant.

However, from a bacteriological point of view, Staphylococci produced more massive infections on Dacron ${ }^{\oplus}$ prostheses, except for the silver-impregnated version. This result also correlated with the grading of clinical infection, with lowest Szilagyi grades for silver-impregnated Dacron ${ }^{\circ}$. In contrast, $E$. coli produced more significant infections on ePTFE grafts, with silver-impregnated Dacron ${ }^{\circledR}$ being again on the lower scale of CFUs/ $\mathrm{ml}$.

The bacteriological results need to be linked to the morpho-pathological analysis, which shows poorer behaviour of the silver coated graft from points of view of tissue incorporation and healing characteristics, comparative to ePTFE graft, which exhibits the best structural performance.

\section{Conclusions}

Silver-impregnation has inhibited bacterial growth in case of E.coli and MSSA infections, but not in MRSA infections.

In case of MRSA infections the ePTFE graft was comparable to the silver-coated graft.

So the silver coated graft is superior to woven and knitted Dacron grafts, but no significantly superior to ePTFE graft.

\section{Acknowledgements}

Research reported in this publication was funded by the European Social Fund, within the framework of the Sectoral Operational Programme Human Resources Development 2007-2013, under award number: POSDRU/19/1.5/S/136893.
Any opinions, findings, and conclusions or recommendations expressed in this material are those of the Authors and do not necessarily reflect the views of the institutions mentioned throughout the manuscript.

We thank our colleagues from the University of Medicine and Pharmacy of Târgu Mureș who provided insight and expertise that greatly assisted the research, although they may not agree with all of the interpretations/conclusions of this paper.

We thank Edit Székely, PhD, MD, Lecturer, Microbiology Department and Anca Delia Mare, PhD, MD, Lecturer, Microbiology Department, for assistance with providing the bacterial strains used in the research.

We would also like to show our gratitude to Marius Petrișor, PhD, MD, Assistant Lecturer, Medical Informatics and Biostatistics Department, for the statistical interpretations and comments that greatly improved the manuscript.

The Author would also like to convey thanks to the University for providing the laboratory and research facilities.

\section{Conflict of interest}

There are no conflicts of interest to declare regarding any of the following: employment, consultancies, stock ownership, equity interests, and patent-licensing arrangements.

\section{References}

1. McCollum CN, Kester RC, Rajah SM, et al. Arterial graft maturation, the duration of thrombotic activity in Dacron aortobifemoral grafts measured by platelet and fibrinogen kinetics. Br J Surg. 1981;68:61.

2. King M, Blais P, Guidoin R, et al. Polyethylene terephthalate (Dacron) vascular prostheses: Material and fabric construction aspects. In Williams DF (ed): Biocompatibility of clinical Implant Materials. CRC Series in Biocompatibility. Boca Raton, Fla. CRC Press. 1981;177.

3. Clowes AW, Gown AM, Hanson Sr, Reidy MA. Mechanisms of arterial graft failure: 1. Role of cellular proliferation in early healing of PTFE prostheses. Am J Pathol. 1985;118:43.

4. Sottiurai VS, Yao JS, Flinn WR, Batson RC. Intimal hyperplasia and neointima: An ultrastructural analysis of thrombosed grafts in humans. Surgery. 1983;93:809.

5. Devine C, Hons B, McCollum C. Heparin bonded Dacron or polytetrafluorethylene for femoropopliteal bypass grafting: A multicenter trial. J Vasc Surg. 2001;33:533.

6. Hernández-Richter T, Schardey HM, Wittmann F, et al. Rifampin and Triclosan but not Silver is Effective in Preventing Bacterial Infection of Vascular Dacron Graft Material. Eur J Vasc Endovasc Surg. 2003;26:550557.

7. Ricco JB, Assadian A, Schneider F, Assadian O. In vitro evaluation of the antimicrobial efficacy of a new silver-triclosan vs a silver collagen-coated polyester vascular graft against methicillin-resistant Staphylococcus aureus. J Vasc Surg. 2012;55(3):823-829.

8. Schneider F, O'Connor S, Becquemin JP. Efficacy of collagen silvercoated polyester and rifampin-soaked vascular grafts to resist infection from MRSA and Escherichia coli in a dog model. Ann Vasc Surg. 2008;22(6):815-821.

9. Eberhart A, Zhang Z, Guidoin R, et al. A new generation of polyurethane vascular prostheses: Rara avis or ignis fatuus? J Biomed Mater Res. 1994;28:1187.

10. Ziegler T, Nerem RM. Tissue engineering a blood vessel, regulation of vascular biology by mechanical stresses. J Cell Biochem. 1994;56:204.

11. Dunn PF, Newman KD, Jones $M$, et al. Seeding of vascular grafts with genetically modified endothelial cells, secretion of recombinant tPA results in decreased seeded cell retention in vitro and in vivo. Circulation. 1996;93:1439.

12. Nunn DB. Structural failure of Dacron arterial grafts. Semin Vasc Surg. 1999;12:83.

13. Homer-Vanniasinkam S. Surgical site and vascular infections: treatment 
and prophylaxis. International Journal of Infectious Diseases 2007;11:(S1) S17-S22.

14. Goldstone J, Moore WS. Infection in vascular prosthesis. Clinical manifestations and surgical management. Am J Surg. 1974;128:225.

15. Chervu A, Moore Ws, Chvapil M, Hendersson T. Efficacy and duration of antistaphylococcal activity comparing three antibiotics bonded to dacron vascular grafts with a collagen release system. J Vasc Surg. 1991;13:897-901.

16. Kaiser AB, Clayson KR, Mulherin Jlet al. Antibiotic prophylaxis in vascular surgery. Ann Surg. 1978;188:283.

17. Kinney EV, Bandyk DF, Seabrook GA, Kelly HM, Towne JB. Antibioticbonded PTFE vascular grafts: the effect of silver antibiotic on bioactivity following implantation. J Surg Res. 1991;50:430-435.

18. Shah PM, Modak S, Fox CL, et al. PTFE graft treated with silver norfloxacin (AgNF): Drug retention and resistance to bacterial challenge.
J Surg Res. 1987;42:298-302.

19. Benvenisty Al, Tannenbaum G, Ahlborn TN, et al. Control of prosthetic bacterial infection: Evaluation of an easily incorporated, tightly bound silver antibiotic PTFE graft. J Surg Res. 1988;44:1-7.

20. Illingworth B, Tweden K, Schroeder R, Cameron JD. In vivo efficacy of silver coated (Silzone) infection-resistant polyester fabric against a biofilm producing bacteria, Staphylococcus epidermidis. J Heart valve Dis. 1998;7:524-530.

21. Collin GR. Decreasing catheter colonization through the use of an antiseptic-impregnated catheter. Chest. 1999;115:1632-1640.

22. Goëau-Brissonnière $O A$, Fabre $D$, Leflon-Guibout V, et al. Comparison of the resistance to infection of rifampin-bonded gelatin-sealed and silver/ collagen-coated polyester prostheses. J Vasc Surg. 2002;36(6):12601263. 